\title{
Implications of $\mathrm{kHz}$ QPOs for the Spin Frequencies and Magnetic Fields of Neutron Stars: New Results from Circinus X-1
}

\author{
Stratos Boutloukos * and Frederick K. Lamb ${ }^{*, \dagger}$ \\ ${ }^{*}$ Center for Theoretical Astrophysics and Department of Physics, University of Illinois, $1110 \mathrm{~W}$. Green St., \\ Urbana, IL 61801, USA \\ ${ }^{\dagger}$ Also, Department of Astronomy
}

\begin{abstract}
Detection of paired kilohertz quasi-periodic oscillations (kHz QPOs) in the X-ray emission of a compact object is compelling evidence that the object is an accreting neutron star. In many neutron stars, the stellar spin rate is equal or roughly equal to $\Delta v$, the frequency separation of the QPO pair, or to $2 \Delta v$. Hence, if the mechanism that produces the kilohertz QPOs is similar in all stars, measurement of $\Delta v$ can provide an estimate of the star's spin rate. The involvement of the stellar spin in producing $\Delta v$ indicates that the magnetic fields of these stars are dynamically important.

We focus here on the implications of the paired $\mathrm{kHz}$ QPOs recently discovered in the low-mass X-ray binary (LMXB) system Cir X-1 [3]. The kHz QPOs discovered in Cir X-1 are generally similar to those seen in other stars, establishing that the compact object in the Cir X-1 system is a neutron star. However, the frequency $v_{u}$ of its upper kHz QPO is up to a factor of three smaller than is typical, and $\Delta v$ varies by about a factor $2(167 \mathrm{~Hz}$, the largest variation so far observed). Periodic oscillations have not yet been detected from Cir X-1, so its spin rate has not yet been measured directly. The low values of $v_{u}$ and the large variation of $\Delta v$ challenge current models of the generation of $\mathrm{kHz}$ QPOs. Improving our understanding of Cir X-1 will improve our knowledge of the spin rates and magnetic fields of all neutron stars.
\end{abstract}

Keywords: low-mass X-ray binaries, millisecond X-ray pulsars, kilohertz quasi-periodic oscillations, Circinus X-1 PACS: $97.10 . \mathrm{Gz}, 97.80 . \mathrm{Jp}, 97.60 . \mathrm{Jd}$

\section{CURRENT UNDERSTANDING}

Power density spectra of the X-ray brightness of LMXBs reveal various types of QPOs. Some two dozen of the 187 known LMXBs [19] have been observed to produce simultaneously two QPOs with frequencies $\sim 100-1300$ $\mathrm{Hz}$ that increase and decrease together by hundreds of $\mathrm{Hz}$ [28]. Some of the key observational features of these $\mathrm{kHz}$ QPOs and their connection to our current theoretical understanding can be summarized as follows [16]:

1. The frequency of one of the two kHz QPOs probably reflects the azimuthal frequency of gas in a streamline at a particular radius in the inner disk. The frequencies of the $\mathrm{kHz}$ QPOs are similar to orbital frequencies near a neutron star and vary by hundreds of Hertz on time scales as short as minutes. Such large, rapid variations are possible if the frequency is related to the orbital frequency at a radius that varies. This can be used to constrain the equation of state of neutron stars [26].

2. The star's spin appears to be involved in producing the frequency separation $\Delta v$ of the two kilohertz QPOs in a pair. This is evident in the millisecond pulsars (MSPs) XTE J1807-294, where $\Delta v \approx v_{s}$ [18], and SAX J1808.4-3658, where $\Delta v \approx v_{s} / 2$ [40]. It is strongly indicated in the other kilohertz
QPO sources, because in all cases where both $\Delta v$ and $v_{s}$ have been measured, the largest value of $\Delta v$ is consistent or approximately consistent with either $v_{s}$ or $v_{s} / 2[34]$. Thus, if paired $\mathrm{kHz}$ QPOs are detected but accretion- and nuclear-powered X-ray oscillations are not, a rough estimate of the star's spin rate can be made using $\Delta v$.

3. The spin rate may be communicated directly by the star's magnetic field or indirectly, e.g., via an anisotropic radiation pattern that rotates with the star. In eihter case the star's magnetic field must be dynamically important.

4. In order to explain the oscillation amplitudes observed, the X-rays must be coming from the surface of the neutron star, because the energy available at larger radii is far too small. A viable model should explain (a) what determines the frequency (radius) at which the principal QPO is generated; (b) why the width of a $\mathrm{kHz}$ QPO peak (range of orbital radii) is so small; (c) how the frequency of the principal QPO changes by factors $\sim 2-3$; and (d) how the spin frequency of the neutron star is involved. So far, the only model that may explain these key features of the kHz QPOs and their amplitudes, energydependence, and dependence on the mass accretion rate is the sonic-point spin-resonance model [17]. 


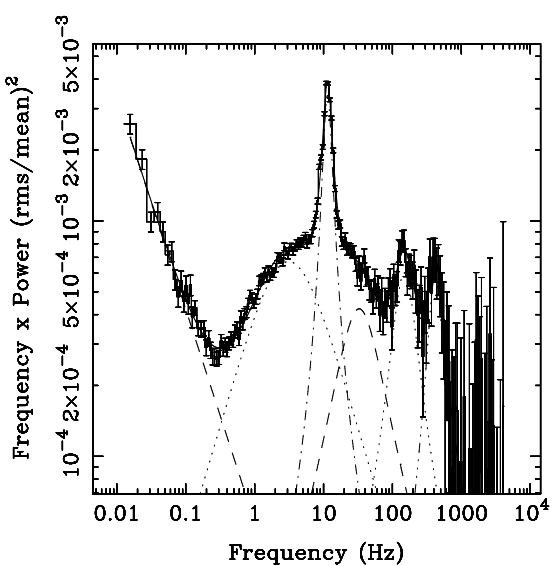

FIGURE 1. Spectral energy density of variations in the Xray brightness of Cir X-1, from an archived RXTE observation. Right: 136 and $404 \mathrm{~Hz}$ "kHz" QPOs; middle: low-frequency QPO; left: very low frequency noise [3].

\section{DISCOVERY OF PAIRED KHZ QPOS IN CIR X-1}

Boutloukos et al. [3, 4] discovered a kHz QPO pair in 11 archived RXTE observations of Cir X-1 and a single $\mathrm{kHz}$ QPO in 69 other observations. Figure 1 shows results from one of these observations. The two $\mathrm{kHz}$ QPOs are visible at $136 \pm 5 \mathrm{~Hz}$ and $404 \pm 2 \mathrm{~Hz}$. Their frequencies track one another and the frequency of the low-frequency QPO in the same way as in other NS LMXBs (see Fig.2). This, and the fact that the frequencies and frequency ratio of the $\mathrm{kHz}$ QPOs vary greatly from one observation to another demonstrates that the Cir X-1 system:

a) contains a NS, resolving a debate that had continued for more than two decades

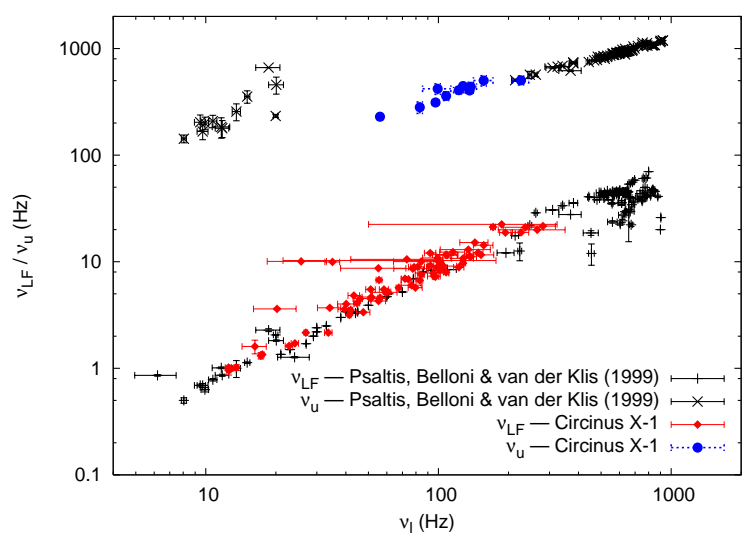

FIGURE 2. Frequencies of the upper $\mathrm{kHz}$ QPO (top) and the low-frequency QPO (bottom) of Cir X-1 and other NS LMXBs, plotted against the frequency of the lower kHz QPO. From [28].
TABLE 1. Accretion- and nuclear-powered millisecond (spin period $P_{S}<10 \mathrm{~ms}$ ) pulsars and EXO 0748-676.

\begin{tabular}{rlll}
$v_{\text {spin }}(\mathrm{Hz})^{*}$ & Object & Reference \\
\hline 1122 & NK & XTE J1739-285 & {$[11]$} \\
619 & NK & 4U 1608-52 & {$[10]$} \\
611 & N & GS 1826-238 & {$[33]$} \\
601 & NK & SAX J1750.8-2900 & {$[12]$} \\
598 & A & IGR J00291+5934 & {$[24]$} \\
589 & N & X 1743-29 & {$[30]$} \\
581 & NK & 4U 1636-53 & {$[42,38,32]$} \\
567 & N & MXB 1659-298 & {$[39]$} \\
550 & ANK & Aql X-1 & {$[5,41]$} \\
530 & N & A 1744-361 & {$[2]$} \\
524 & NK & KS 1731-260 & {$[29]$} \\
442 & AN & SAX J1748.9-2021 & {$[9,1,13]$} \\
435 & A & XTE J1751-305 & {$[25]$} \\
401 & ANK & SAX J1808.4-3658 & {$[37,6]$} \\
377 & A & HETE J1900.1-2455 & {$[27]$} \\
363 & NK & 4U 1728-34 & {$[31]$} \\
330 & NK & 4U 1702-429 & {$[22]$} \\
314 & AN & XTE J1814-338 & {$[23]$} \\
294 & NK & IGR J17191-2821 & {$[20,14]$} \\
270 & N & 4U 1916-05 & {$[8]$} \\
191 & AK & XTE J1807.4-294 & {$[21,36]$} \\
185 & A & XTE J0929-314 & {$[7]$} \\
182 & A & SWIFT J1756.9-2508 & {$[15]$} \\
45 & N & EXO 0748-676 & {$[35]$} \\
\hline & & &
\end{tabular}

* Spin frequency inferred from periodic or nearly periodic Xray oscillations. A: accretion-powered millisecond pulsar. $\mathrm{N}$ : nuclear-powered millisecond pulsar. K: kilohertz QPO source.

b) has a dynamically important magnetic field, as inferred from the current understanding of how the $\mathrm{kHz}$ QPOs are generated, and

c) has a spin frequency of several hundred $\mathrm{Hz}$, based on the frequency separation of the $\mathrm{kHz} \mathrm{QPO}$ pair.

Table 11 shows the inferred spin rates of EXO 0748-676 and the 23 known accreting millisecond pulsars. Nuclear- and accretion-powered oscillations have been detected in 17 and 10 of them, respectively; $\mathrm{kHz}$ QPOs have been detected in 11 MSPs.

\section{UNIQUE PROPERTIES OF THE PAIRED KHZ QPOS SEEN IN CIR X-1}

The discovery of paired kHz QPOs in Cir X-1 has answered several important questions, but has also opened new ones:

- The frequencies of the paired $\mathrm{kHz}$ QPOs seen in Cir X-1 reach the lowest values so far observed in NS LMXBs: $55 \mathrm{~Hz}$ for the lower QPO and 250 $\mathrm{Hz}$ for the upper QPO. These observed frequencies extend the previously known frequency correlation 


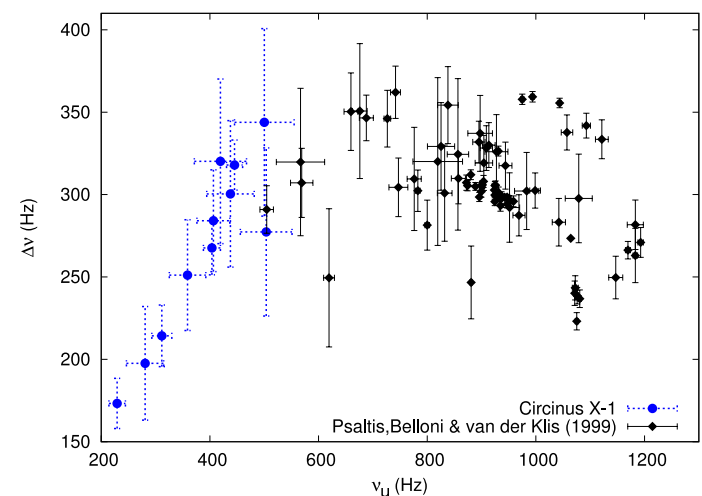

FIGURE 3. Frequency separation of $\mathrm{kHz}$ QPO pairs vs. frequency of the upper kHz QPO, for the same sources as in Fig.2

([28], Fig. 26) over a range of a factor 4 ; single $\mathrm{kHz}$ QPOs were seen with frequencies as low as $10 \mathrm{~Hz}$. According to the current theoretical understanding . (see above), this implies that the frequency of the principal $\mathrm{kHz}$ QPO is sometimes generated at a radius $\sim 50 \mathrm{~km}$, for a $2 \mathrm{M}_{\odot}$ star.

- The frequency separation $\Delta v$ of the $\mathrm{kHz}$ QPOs reaches the lowest value so far observed $(170 \mathrm{~Hz})$, and $\Delta v / v_{u}$ is $0.55-0.75$, larger than the values $\sim 0.2-0.6$ observed in other $\mathrm{kHz}$ QPO systems.

- The frequency separation varies by $\sim 180 \mathrm{~Hz}$ (more than a factor 2), more than the variation of the frequency separation among all other known systems (see Fig. 3).

- The frequency separation increases systematically with increasing upper $\mathrm{kHz}$ QPO frequency, unlike in other $\mathrm{kHz} \mathrm{QPO}$ sources.

The $\mathrm{kHz}$ QPOs discovered in Cir X-1 challenge our current theoretical understanding of how the $\mathrm{kHz}$ QPOs are generated. Understanding them will improve our understanding of how the $\mathrm{kHz}$ QPOs are generated, enhancing our ability to determine the masses, spin rates, and magnetic field of the neutron stars in LMXBs.

\section{ACKNOWLEDGMENTS}

These results are based on research supported by NSF Award numbers AST 00-98399 and AST 07-09015 and NASA Award number NAG 5-12030

\section{REFERENCES}

1. D. Altamirano et al., ApJL submitted (2007), arXiv:0708.1316

2. S. Bhattacharyya et al., ApJ 639, L31 (2006)
3. S. Boutloukos et al., ApJ 653, 1435 (2006)

4. S. Boutloukos et al., ApJ 694, 596 (2007)

5. P. Casella et al., ApJ, submitted (2007), arXiv:0708.1110

6. D. Chakrabarty and E.H. Morgan, Nature 394, 346 (1998)

7. D.K. Galloway et al., ApJ 576, L137 (2002)

8. D.K. Galloway et al., ApJ 549, L85 (2001)

9. F. Gavriil et al., ApJL submitted (2007), arXiv:0708.0829

10. J.M. Hartman et al., "Discovery of $619 \mathrm{~Hz}$ Thermonuclear Burst Oscillations in the Low-Mass X-Ray Binary $4 \mathrm{U}$ 1608-52", in HEAD meeting 7, Bulletin of the American Astronomical Society Vol. 35, 2003, p.865

11. P. Kaaret et al., ApJ 657, 97 (2007)

12. P. Kaaret et al., ApJ 575, 1018 (2002)

13. P. Kaaret et al., ApJ 598, 481 (2003)

14. M. Klein-Wolt et al., ATel 1075 (2007)

15. H.A. Krimm et al. (2007), ApJL accepted (2007), arXiv:0709.1693

16. F.K. Lamb and S. Boutloukos, "Accreting Neutron Stars in Low-Mass X-Ray Binary Systems", to appear in Short Period Binaries, edited by E.F. Milone, D.A. Leahy, and D. Hobil, Springer, Dordrecht, 2007, arXiv:0705.0155

17. F.K. Lamb and M.C. Miller, ApJ submitted (2007), astro-ph/0308179

18. M. Linares et al., ApJ 634, 1250 (2005)

19. Q.Z. Liu, J. van Paradijs, and E.P.J. van den Heuvel, $A \& A$ 469, 807 (2007)

20. C.B. Markwardt et al., ATel 1068 (2007)

21. C.B. Markwardt, E. Smith, and J.H. Swank, IAU Circ. 8080, 2 (2003)

22. C.B. Markwardt, T.E. Strohmayer, and J.H. Swank, ApJ 512, L125 (1999)

23. C.B. Markwardt and J.H. Swank, IAU Circ. 8144, 1 (2003)

24. C.B. Markwardt, J.H. Swank, and T.E. Strohmayer, ATel 353, 1 (2004)

25. C.B. Markwardt et al., ApJ 575, L21 (2002)

26. M.C. Miller, F.K. Lamb, and D. Psaltis, ApJ 508, 791 (1998)

27. E. Morgan, P. Kaaret, and R. Vanderspek, ATel 523 (2005)

28. D. Psaltis, T. Belloni, and M. van der Klis, ApJ 520, 262 (1999)

29. D.A. Smith, E.H. Morgan, and H. Bradt: ApJ 482, L65 (1997)

30. T.E. Strohmayer et al., ApJ 486, 355 (1997)

31. T.E. Strohmayer et al., ApJ 469, L9 (1996)

32. T.E. Strohmayer et al., ApJ 498, L135 (1998)

33. T.W.J. Thompson et al., ApJ 634, 1261 (2005)

34. M. van der Klis, "Rapid X-ray Variability", in Compact Stellar X-ray Sources, edited by W. Lewin and M. van der Klis, Cambridge University Press, Cambridge, UK, 2006, p. 39, astro-ph/0410551

35. A.R. Villarreal and T.E. Strohmayer, ApJ 614, L121 (2004)

36. R. Wijnands, "Accretion-Driven Millisecond X-ray Pulsars", in Trends in Pulsar Research, edited by J.A. Lowry, Nova Science Publishers Inc., New York, 2006, p.53, astro-ph/0501264

37. R. Wijnands and M. van der Klis: Nature 394, 344 (1998)

38. R.A.D. Wijnands et al., ApJ 479, L14 (1997)

39. R. Wijnands, T. Strohmayer, and L.M. Franco, ApJ 549, L71 (2001)

40. R. Wijnands et al., Nature 424, 44 (2003)

41. W. Zhang et al., ApJ 495, L9 (1998)

42. W. Zhang et al., IAU Circ. 6541, 1 (1997) 